\title{
PRESENT AND FUTURE HIGH-ENERGY ACCELERATORS FOR NEUTRINO EXPERIMENTS
}

\author{
I. Kourbanis ${ }^{\#}$, FERMILAB*, Batavia, IL 60510, U.S.A.
}

\section{Abstract}

There is an active neutrino program making use of the high-energy (larger than $50 \mathrm{GeV}$ ) accelerators both in USA at Fermilab with NuMI and at CERN in Europe with CNGS. In this paper we will review the prospects for high intensity high energy beams in those two locations during the next decade.

\section{THE CERN NEUTRINO PROGRAM}

The CNGS neutrino beam has been optimized for the $v_{\mu} \rightarrow v_{\tau}$ appearance search. The beam protons from the SPS are extracted with energies of $400 \mathrm{GeV}$ hitting a graphite target $830 \mathrm{~m}$ from SPS. After a magnetic horn system for focusing the pions, a decay pipe of $1000 \mathrm{~m}$ follows. The beam-line design was accomplished on the basis of the previous experience with the WANF beam at CERN SPS [1,2].

\section{CERN Accelerator Complex}

The CERN proton accelerators that are used for CNGS are shown in Fig. 1 and are:

- Linac 2

- Proton Synchrotron Booster-PSB

- Proton Synchrotron-PS

- Super Proton Synchrotron-SPS

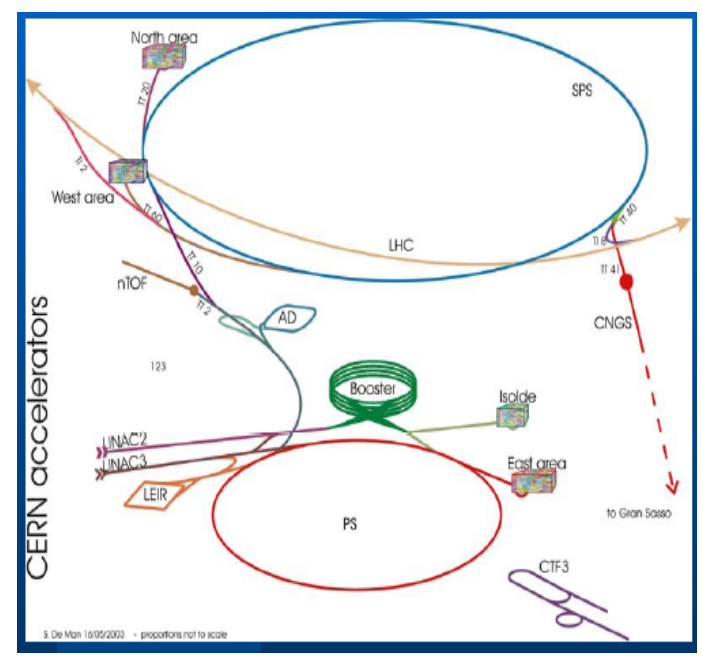

Figure 1: CERN Proton accelerators for CNGS.

The injection and extraction energies of the CERN proton accelerators along with their commissioning year are shown in Table1 (from [3]).

\footnotetext{
* Operated by Fermi Research Alliance, LLC under Contact No. DEAC02-07CH11359 with the United States Department of Energy.

\#ioanis@fnal.gov
}

Table 1: Table of the CERN accelerators used for CNGS with their inj./extr. energies and their year of commissioning

\begin{tabular}{|l|c|c|c|}
\hline Accelerator & $\begin{array}{c}\text { Inj. Kinetic Energy } \\
{[\mathrm{GeV}]}\end{array}$ & $\begin{array}{c}\text { Ext. Kinetic Energy } \\
{[\mathrm{GeV}]}\end{array}$ & $\begin{array}{c}\text { Commissioning } \\
\text { year }\end{array}$ \\
\hline Linac 2 & 0.00075 & 0.05 & 1979 \\
\hline PSB & 0.05 & 1.4 & 1972 \\
\hline PS & 1.4 & 13.1 & 1959 \\
\hline SPS & 13.1 & 399.1 & 1976 \\
\hline
\end{tabular}

As can be seen from table 1, most of the CERN proton accelerators are already more than 30 years old and are the same accelerators that are going to be used as LHC injectors. The nominal LHC beam has a much higher (x10) bunch intensity than the nominal CNGS beam but lower total beam intensity.

\section{Accelerator Upgrades}

In order to prepare for the high intensity LHC beam the CERN accelerator chain has been upgraded [4]. Some of the upgrades/modifications included:

- In the Booster acceleration at harmonic $\mathrm{h}=1$ replaced the $\mathrm{h}=5$ used in the past in order to help the coupled bunch instabilities.

- In the PS the injection energy was increased from 1 $\mathrm{GeV}$ to $1.4 \mathrm{GeV}$ to reduce space charge tune spread.

- In the SPS the longitudinal impedance was greatly reduced by shielding 800 vacuum ports along with kickers and septa. This eliminated the microwave instability greatly reducing the longitudinal emittance blow-up during acceleration.

- The equipment used for leptons were removed from all machines. This included a $114 \mathrm{MHz}$ RF system in the PS and three RF systems in the SPS.

\section{High Intensity SPS operation for CNGS}

In 2004, during a 3 week beam test a maximum intensity of 5.3E13 protons was obtained at $400 \mathrm{GeV}$ in a CNGS cycle. The main limitation for further intensity increase was the beam loss [4].

Typical beam intensities in the different machines are shown in Table 2. The beam intensity in the SPS along with the energy ramp is shown in Fig. 2. It takes two PS injections $1.2 \mathrm{sec}$ apart to fill the SPS for CNGS. Unlike the LHC beam, this beam crosses transition energy, not only in the PS but also in the SPS. 
Table 2: Beam transmission through CERN accelerator chain for the high intensity SPS run CNGS (From [4]).

\begin{tabular}{|l|l|l|l|l|}
\hline Accelerator & $\begin{array}{l}\text { Injected } \\
\text { Intensity } \\
(\mathrm{E} 13)\end{array}$ & $\begin{array}{l}\text { Accelerated } \\
\text { Intensity } \\
(\mathrm{E} 13)\end{array}$ & $\begin{array}{l}\text { Extracted } \\
\text { Intensity } \\
(\mathrm{E} 13)\end{array}$ & $\begin{array}{l}\text { Acc. } \\
\text { Eff. } \\
(\%)\end{array}$ \\
\hline Booster & 4.3 & 3.84 & 3.65 & 84.9 \\
PS & 3.57 & 3.42 & 3.15 & 88.2 \\
SPS & $3.0 \times 2$ & 5.7 after tr. & ------- & \\
SPS & $2.9 \times 2$ & 5.5 after tr. & 5.3 & 91.3 \\
\hline
\end{tabular}

With the present injectors, it was demonstrated that a maximum intensity of $3 \times 10^{13}$ protons can be injected into the SPS every $1.2 \mathrm{sec}$. Taking into account beam losses, this could lead to a total intensity of $5.7 \times 10^{13}$ protons at $400 \mathrm{GeV}$ in the SPS. This corresponds to a beam power of $608 \mathrm{KW}$ (assuming a 6 sec SPS cycle).

The present $\mathrm{rf}$ system in the SPS limits the beam intensity that can be accelerated to about $6 \times 10^{13}$ protons/cycle. The CNGS target limits the SPS intensity to $2 \times 3.5 \times 10^{13}$ pot per $6 \mathrm{sec}$ cycle ("ultimate intensity").

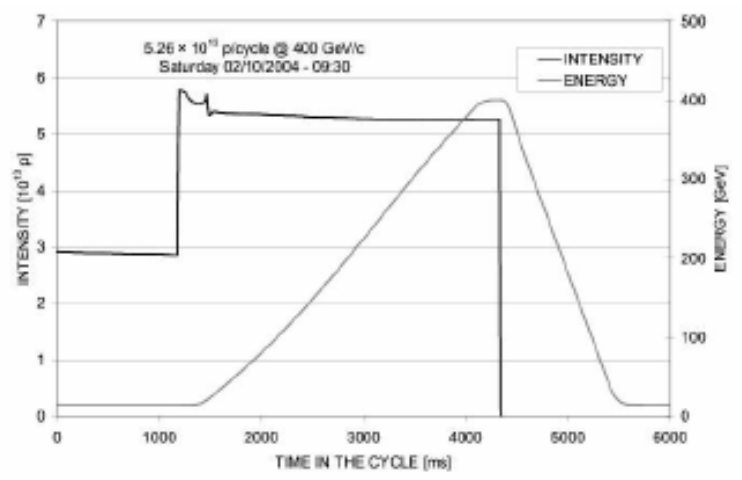

Figure 2: Beam transmission in the SPS at the end of the high intensity run. The sharp loss during acceleration is happening during transition crossing.

\section{Addressing the beam loss limitations}

As it can be seen in Table 2 the overall efficiency for the record SPS intensities taking into account and the loss during transfers is about $62 \%$. In order to be able to run at his high intensities the beam loss especially at the high energies must be minimized. The 3 most important losses along with proposed solutions are outlined below.

- Losses at extraction in the PS. Plan to reduce these losses by changing the present multi-turn extraction (transverse slicing of the PS beam in the horizontal plane by a thin electrostatic septum) with a novel scheme using resonant islands [6].

- Low energy losses in the SPS. Those losses happen at injection, at the injection front porch and during transition crossing. These losses will be reduced by opening the physical vertical aperture of the SPS, and modifying the rf beam control to reduce transient beam loading during transition crossing.

- SPS extraction losses. The cause of these losses is beam in the extraction kicker gap. The beam in the gap is originated at injection due to the poor capture of the injected batches and the recapture of particles at the start of ramp. A new beam control for the separate capture of each batch and cleaning of the beam gap at low energy with the transverse damper is expected to greatly improve those losses.

\section{Total number of protons with the present Injectors}

The total number of protons to the CNGS target depends not only on the peak beam intensity but also on the beam availability and the beam sharing with other users. For 200 days $\left(1.7 \times 10^{7} \mathrm{sec}\right)$ of operation with $80 \%$ machine efficiency and with $45 \%$ of the SPS time available for CNGS, we obtain $5.9 \times 10^{19}$ pot per year for an intensity of $5.7 \times 10^{13}$ protons at $400 \mathrm{GeV}$. If no other user of the SPS is scheduled (except LHC) the SPS time available for SPS can be as high as $85 \%$ and the number of protons per year can be as high as $1.11 \times 10^{20}$. It should be noted here that the CNGS neutrino components were designed to tolerate up to $1.38 \times 10^{20}$ pot for 5 years [5].

\section{Proton flux for CNGS with new injectors}

In order to maximize the LHC performance, most of the CERN injectors are to be replaced with new higher performance accelerators [5,7].

In the first stage, a new linac, Linac4 is built, injecting protons at $160 \mathrm{MeV}$ thus halving the space charge effects in PSB.

In the second stage, the PS is replaced by PS2, a new 50 $\mathrm{GeV}$ synchrotron. The PS2 will be double the size of the PS and will be able to accelerate up to $1.1 \times 10^{14}$ protons per pulse $(3.6 \mathrm{sec})$. Because of the size of PS2 its injection energy has to be higher than $3.5 \mathrm{GeV}$ so as an intermediate solution the PS will be used as an injector till a $3.5-4 \mathrm{GeV}$ superconducting linac the SPL is built. This will limit PS2 performance because of space charge limitations in the PS and because of cycling and filling constraints between the two machines.

With the new injector PS2, the length of the SPS cycle for CNGS can be reduced to 4.8 sec. A total of $1.0 \times 10^{14}$ protons can be accelerated to $400 \mathrm{GeV}$ in the SPS for a total beam power of $1.3 \mathrm{MW}$. The total number of protons per year can reach $2.4 \times 10^{20}$ pot assuming $85 \%$ SPS availability. A new rf system will be needed in the SPS to accelerate this beam intensity. It should also be noted that the CNGS facility will also need a re-design and a major rebuild.

The protons on the CNGS target for the different scenarios are shown in Table 3 [5]. For all the scenarios 200 days of operation with $80 \%$ machine availability is assumed. 
Table 3: Protons on target per year (E19) to CNGS for different scenarios.

\begin{tabular}{|l|l|c|c|c|c|}
\hline & SPS cycle length & \multicolumn{2}{|c|}{$6 \mathrm{sec}$} & \multicolumn{2}{c|}{$4.8 \mathrm{sec}$} \\
\cline { 2 - 6 } & Injection momentum & \multicolumn{2}{|c|}{$14 \mathrm{GeV}$} & \multicolumn{2}{c|}{$26 \mathrm{GeV}$} \\
\hline & $\begin{array}{l}\text { Max SPS } \\
\text { Intensity @ 400 sharing } \\
\text { GeV (E13) }\end{array}$ & 0.45 & 0.85 & 0.45 & 0.85 \\
\hline $\begin{array}{l}\text { Present injectors + } \\
\text { machines" } \\
\text { improvements }\end{array}$ & $\begin{array}{l}\text { 4.8-"Nominal } \\
\text { CNGS" }\end{array}$ & 5 & 9.4 & & \\
\cline { 2 - 6 } & $\mathbf{5 . 7 - " M a x . ~ S P S " ~}$ & 5.9 & 11.1 & & \\
\hline $\begin{array}{l}\text { Future injectors } \\
\text { +SPS RF Upgrade }\end{array}$ & $7-$-Ultimate CNGS" & & & 9 & 17.1 \\
\hline $\begin{array}{l}\text { Future injectors } \\
\text { +new SPS RF system } \\
\text { +CNGS new } \\
\text { equipment design }\end{array}$ & 10 -"Max. PS2" & & & 12.9 & 24.5 \\
\hline
\end{tabular}

\section{THE FERMILAB NEUTRINO PROGRAM}

The NuMI neutrino beam has been optimized for neutrino oscillations. The beam protons from Main Injector are extracted at $120 \mathrm{GeV}$ hitting a graphite target $375 \mathrm{~m}$ away. The pions coming out of the target are focused with a system of two magnetic horns and decay in a $675 \mathrm{~m}$ long pipe. The pion focusing of NuMI can be optimized for 3 different neutrino energies by adjusting the locations of the second horn and target with respect to the first horn [8].

\section{Fermilab Accelerator complex}

The Fermilab proton accelerators that are currently used for NuMI are shown in Fig. 3 and are:

- Linac

- Booster accelerator

- Main Injector

There are also shown the two antiproton production and storage rings the Debuncher and the Accumulator along with the newest antiproton storage ring the Recycler. Those rings are to be re-configured at the end of the collider program in order to be used in the neutrino program.

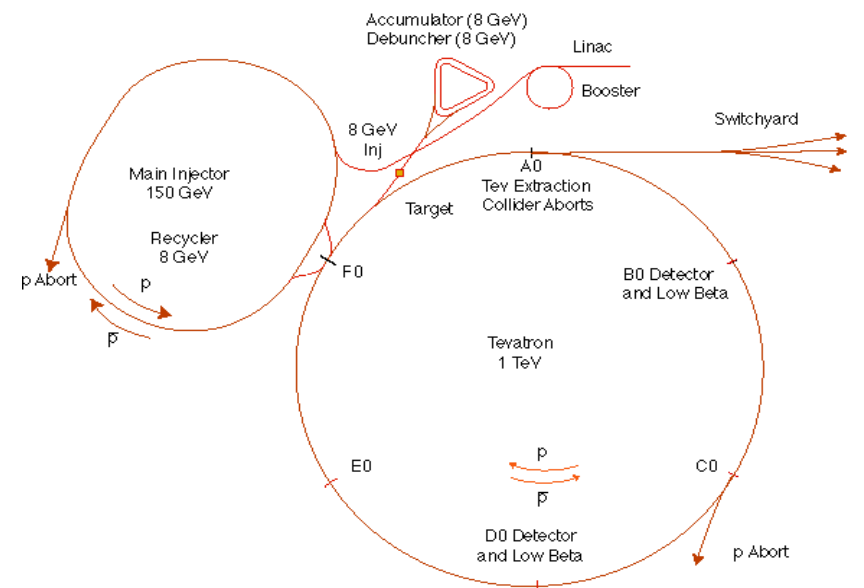

Figure 3: Fermilab Proton accelerators.
The Injection and extraction energies of the Fermilab accelerators along with their commissioning year are shown in Table 6. Except the Main Injector the Fermilab injectors are as old as the CERN accelerators. The high energy part of the linac was upgraded in 1993 raising the extraction energy to $400 \mathrm{MeV}$ from $200 \mathrm{MeV}$.The Booster has been also upgraded with a collimation system and a new injection system.

Table 4: Table of the Fermilab accelerators used for NuMI with their injection/extraction energies and their year of commissioning.

\begin{tabular}{|l|l|l|l|}
\hline Accelerator & $\begin{array}{l}\text { Injection } \\
\text { Kinetic } \\
\text { Energy } \\
{[\mathrm{GeV}]}\end{array}$ & $\begin{array}{l}\text { Extraction } \\
\text { Kinetic } \\
\text { Energy } \\
{[\mathrm{Gev}]}\end{array}$ & $\begin{array}{l}\text { Commissioning } \\
\text { year }\end{array}$ \\
\hline Linac & 0.025 & 0.4 & 1970 \\
\hline Booster & 0.4 & 8 & 1971 \\
\hline $\begin{array}{l}\text { Main } \\
\text { Injector }\end{array}$ & 8 & 119 & 1999 \\
\hline Recycler & 8 & 8 & 2004 \\
\hline $\begin{array}{l}\text { Debuncher/ } \\
\text { Acumulator }\end{array}$ & 8 & 8 & 1986 \\
\hline
\end{tabular}

\section{Current NuMI operation}

The NuMI neutrino beam was commissioned in 2005. Since then more than $3.4 \mathrm{E} 20$ protons have been delivered to the NuMI target.

In the current NuMI operation a total of 7 Booster batches are injected in Main Injector in a mixed mode cycle. First two batches are injected, slipped stacked and recaptured. Then 5 more Booster batches are injected and the whole beam is accelerated to $120 \mathrm{GeV}$. At $120 \mathrm{GeV}$ the slipped stacked beam gets extracted first to the pbar target and then the 5 remaining Booster batches get extracted to the NuMI target. The total Main Injector intensity is around $3.3 \times 10^{13}$ protons per cycle out of which $2.45 \times 10^{13}$ go to the NuMI target. It should be noted that in this stage the repetition rate of the mixed mode cycle is not determined by the Main Injector cycle time $(2.0 \mathrm{sec})$ but by the antiproton stacking requirements that limit the min. rep rate to $2.2 \mathrm{sec}$ or larger.

For the proton projections to the NuMI target a total of 308 days $\left(2.7 \times 10^{7} \mathrm{sec}\right)$ of running a year is assumed with $61 \%$ overall efficiency (85\% complex uptime, $90 \%$ average to peak correction and $80 \%$ NuMI uptime) to get 1.7E20 protons per year.

\section{Upgrades for higher neutrino power}

All the Fermilab upgrades for higher neutrino power take advantage of .the Main Injector capabilities:

- Large transverse acceptance $(40 \pi-\mathrm{mm}-\mathrm{mrad})$ and momentum aperture (1.7\%).

- Fast cycle time $(1.5 \mathrm{sec})$

- RF system that can be upgraded to accelerate more beam power. 
Three stages of upgrades are envisioned that are known as the "Proton Plan", the "NOvA Project" and the "SNuMI".

\section{"Proton Plan"}

In this first stage of upgrades expected to become operational in 2008, the Main Injector intensity to NuMI is increased by implementing slip stacking for the NuMI batches (multi-batch slip stacking).Ten Booster batches are slipped stacked in Main Injector into five double intensity ones and recaptured. After recapture an additional batch is injected and the whole beam is accelerated to $120 \mathrm{GeV}$. The Main Injector cycle time is increased to $2.2 \mathrm{sec}$ because of the extra injections. The whole process is expected to be $95 \%$ efficient and assuming an intensity of $4.3 \times 10^{12}$ protons per Booster batch, the total Main Injector intensity at $120 \mathrm{GeV}$ is $4.5 \times 10^{13}$ with $3.7 \times 10^{13}$ protons delivered to NuMI target.

The beam power to NuMI will be increased to $320 \mathrm{KW}$ and the total number of protons per year to the NuMI target to $2.8 \times 10^{20}$.

The multi-batch has been successfully demonstrated in Main Injector [9] where a record intensity of $4.6 \times 10^{13}$ protons has been accelerated (Fig. 4). At this stage the managing of losses is the big challenge. A two stage collimation system has been specifically designed to localize the losses from un-captured beam during acceleration [10].The distribution of losses around Main Injector with and without the collimators is shown in Fig. 5. The Main Injector bunch by bunch transverse dampers will be re-configured to work during slip stacking. These dampers will be also used to anti-damp any beam that would end up in the antiproton extraction kicker gap.

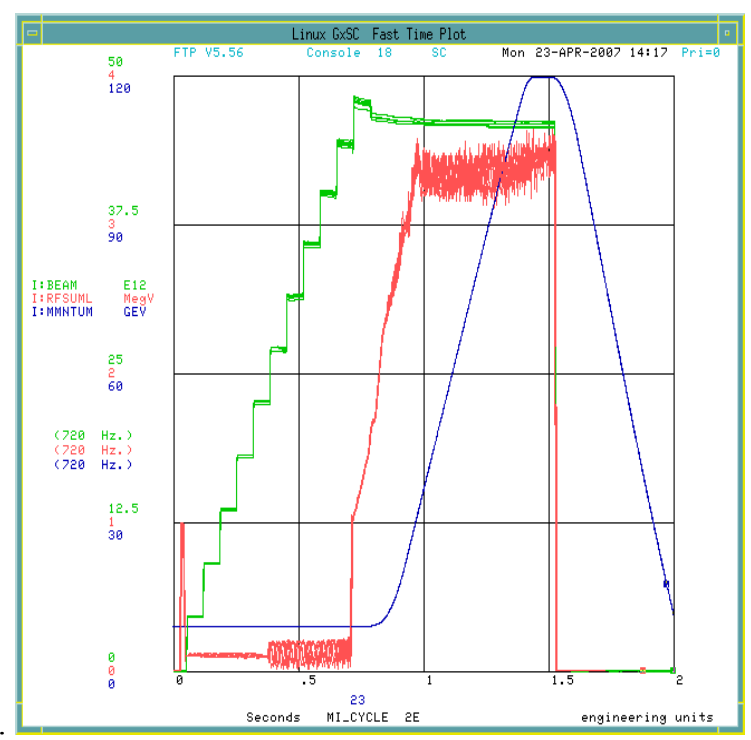

Figure 4: Beam intensity in Main Injector with multibatch slip stacking.
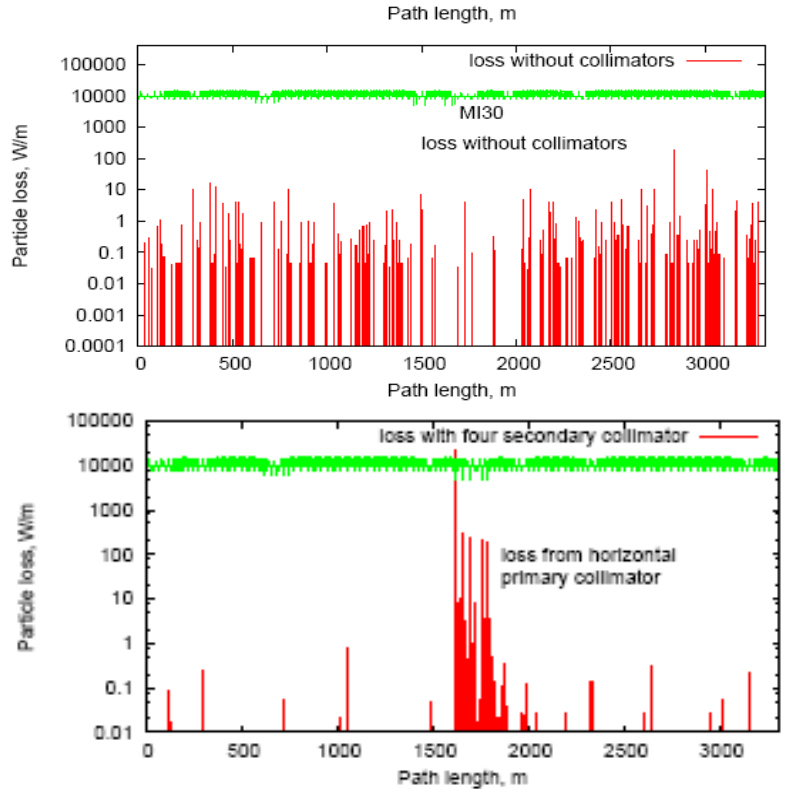

Figure 5: Power loss distribution across Main Injector without (top) and with collimators (bottom) .

\section{The "NOvA Project"}

In this stage planned to happen after the end of the collider run, the Recycler Ring is re-configured from an antiproton storage ring to a proton ring where the multibatch slip stacking is taking place. Since all the beam will be extracted to NuMI up to twelve Booster batches can be slipped stacked in the Recycler. After slip stacking is complete the beam is transferred in the Main Injector where is accelerated to $120 \mathrm{GeV}$. The Main Injector cycle time is now reduced to $1.5 \mathrm{sec}$ since most of the $8 \mathrm{GeV}$ dwell time is eliminated. By installing two extra $\mathrm{rf}$ cavities (from spares) and upgrading the defocusing quad supply we can increase the maximum acceleration rate in the Main Injector ramp from 204 to $240 \mathrm{GeV} / \mathrm{sec}$ and reduce the cycle time to $1.33 \mathrm{sec}$. The beam intensity in the Main Injector is increased by only $9 \%$ to $4.9 \times 10^{13}$ protons while the beam power and the protons per year to the NuMI target are increased by about a factor of 2 to $700 \mathrm{KW}$ and $6.1 \times 10^{20}$ respectively.

A couple of new injection lines need to be build, one from the Booster to the Recycler and one from Recycler to Main Injector. Five new kicker systems will also need to be build for Recycler that also include a gap clearing kicker. This kicker will be used to cleanly abort any beam that has leaked during slip stacking in the injection gap. A new $53 \mathrm{MHz}$ system will be required for slip stacking in Recycler.

\section{The "SNuMI" plan}

The number of Booster batches that is stacked in Recycler cannot be increased any more by using slip stacking because of the larger momentum aperture required and the beam losses. It has been proposed to use momentum stacking in the antiproton Accumulator in 
order to further increase the intensity in Main Injector and to NuMI [11].

The steps in this multi-stage Proton Accumulator scheme are outlined below:

- Momentum stack in the Accumulator. For this step a Booster batch is injected at the high momentum orbit of the Accumulator every $67 \mathrm{msec}$. The new batch is decelerated towards the core orbit and gets merged with existing beam. After momentum stacking of 3 Booster batches the beam is captured and extracted to the Recycler.

- Box Car Stack in the Recycler. The Recycler gets loaded with a new Accumulator batch every 200 msec. A total of six Accumulator batches are placed sequentially around the Recycler.

- Load the Main Injector in a single turn.

The main advantages of the momentum stacking include:

- No transient beam loading effects

- Speed of the process

- Small longitudinal emittance dilution

- No need of transfer cogging in the Booster

For this stage two new beam lines will be needed, one to connect the Booster to Accumulator and another one to connect the Accumulator to the Recycler. Two 7.5 MHz rf systems will be needed, one in the Accumulator for recapturing the beam, and one in the Recycler for accepting the Accumulator beam. Before transferring the Recycler beam to the Main Injector the beam will be recaptured in $53 \mathrm{MHz}$ using the rf system that was installed for slip stacking.

Assuming $4.7 \times 10^{12}$ protons per Booster batch, the Main Injector intensity reaches $8.3 \times 10^{13}$ protons and the beam power to NuMI the $1.2 \mathrm{MW}$. In order to accelerate this higher beam power a second power tube needs to be installed in each of the Main Injector cavities. The installation of a $\gamma_{t}$ system in Main Injector is also planned. The protons per year to NuMI can reach $10 \times 10^{20}$. The SNuMI plan is currently at a conceptual stage and it is not yet officially supported.

The present and future scenarios for NuMI are summarized in Table 5. The upgrades of the NuMI facility required for all the above stages are outlined in [12].

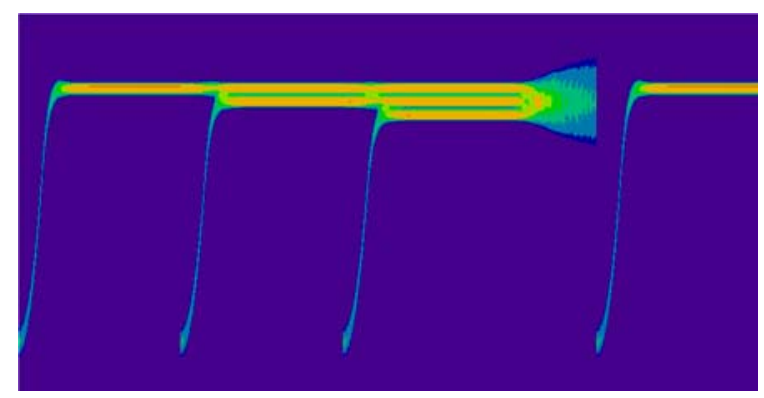

Figure 4: Tracking simulation of the energy profile vs. time for momentum stacking three Booster batches.
Table 5: Present and future operating scenarios for NuMI.

\begin{tabular}{|c|c|c|c|c|}
\hline & $\begin{array}{c}\text { Present } \\
\text { Conditions } \\
\text { Multi-batch slip- } \\
\text { stacking in MII }\end{array}$ & $\begin{array}{c}\text { Proton Plan } \\
\text { Multi-batch } \\
\text { slip-stacking } \\
\text { in MII }\end{array}$ & $\begin{array}{c}\text { NOvA } \\
\text { Multi-batch } \\
\text { slip-stacking in } \\
\text { Recycler }\end{array}$ & \begin{tabular}{|c|} 
SNuVII \\
Momentum Stacking in \\
the Accumulator, boxcar \\
stacking in Recycler
\end{tabular} \\
\hline $\begin{array}{l}\text { Booster intensity } \\
\text { (protons/batch) }\end{array}$ & $4.3-4.5 \times 10^{12}$ & $4.3 \times 10^{12}$ & $4.3 \times 10^{12}$ & $4.7 \times 10^{12}$ \\
\hline $\begin{array}{l}\text { No. Booster } \\
\text { batches }\end{array}$ & 7 & $\rightarrow 11$ & $\rightarrow 12$ & 18 \\
\hline MII cycle time (s) & 2.4 & $\rightarrow 2.2-$ & $\longrightarrow 1.333$ & 1.333 \\
\hline $\begin{array}{l}\text { MII intensity } \\
\text { (ppp) }\end{array}$ & $3.3 \times 10^{13}$ & $4.5 \times 10^{13}$ & $4.9 \times 10^{13}$ & $8.3 \times 10^{13}$ \\
\hline $\begin{array}{l}\text { To anti-proton } \\
\text { source (ppp) }\end{array}$ & $8.8 \times 10^{12}$ & $8.2 \times 10^{12}$ & 0 & 0 \\
\hline To NuMII (ppp) & $2.45 \times 10^{13}$ & $3.7 \times 10^{13}$ & $4.9 \times 10^{13}$ & $8.3 \times 10^{13}$ \\
\hline $\begin{array}{l}\text { NuVII beam } \\
\text { power (kW) }\end{array}$ & 192 & $\rightarrow 320$ & $\rightarrow 700$ & 1200 \\
\hline PoT/yr to NuVII & $2 \times 10^{20}-$ & $\rightarrow 3 \times 10^{20}=$ & $\rightarrow 6 \times 10^{20}$ & $10 \times 10^{20}$ \\
\hline
\end{tabular}

\section{CONCLUSIONS}

There is an active program of upgrades to the high energy accelerators both at CERN and at Fermilab that will raise the beam power available to neutrino experiments to 1.2-1.3MW. The CERN upgrades depend on new injectors while the Fermilab ones depend on the Main Injector and reconfiguration of existing antiproton storage rings.

\section{REFERENCES}

[1] L. Casagrande et al., "The alignment of the CERN West Area neutrino facility",CERN-96-06.

[2] G. Acquistapace et al.,"The CERN neutrino beam to Gran Sasso", CERN 98-02,INFN/AE-98-05 (1998);CERN-SL/99-034(DI),INFN/AE-99/05 Addendum.

[3] G. Arduini., "The CNGS Beam Challenge", Seminar given on May 112005.

[4] E. Shaposhnikova et al., "Recent Intensity Increase in The CERN Accelerator Chain",CERN-AB-2005-029.

[5] M. Meddahi, E. Shaposhnikova, "Analysis of the maximum potential proton flux to CNGS",CERNAB-2007-013.

[6] M. Giovannozzi et al., "The CERN PS multi-turn extraction based on beam splitting in stable islands of transverse phase space", Design Report, CERN-2006011.

[7] M. Benedict et al., "Potential for Neutrino and Radioactive Beam Physics of the Foreseen Upgrades of the CERN Accelerators", Design Report, CERNAB-2006-019-PAF.

[8] The Fermilab NuMI group, "NuMI Facility Technical Design Report", Fermilab Report NuMI-346, 1998.

[9] K. Seiya et al., "Multi-batch Slip Stacking in the Main Injector at Fermilab", These proceedings.

[10]A. Drozhdin et al., "Collimation System Design For Beam Loss Localization With Slip Stacking Injection in The Fermilab Main Injector", These proceedings.

[11] D. McGinnis., "A 2 Megawatt Multi-stage Proton Accumulator", Fermilab Beams Document 1782, Nov. 2005.

[12] M. Martens. et al., "Upgrades to the Fermilab NuMI Beamline", These proceedings. 
